\title{
Radio Observations of HD 80606 Near Planetary Periastron
}

\author{
T. Joseph W. Lazio ${ }^{1,2}$ \\ Naval Research Laboratory, 4555 Overlook Ave. SW, Washington, DC 20375-5351 USA \\ P. D. Shankland \\ U.S. Naval Observatory Flagstaff Station, Flagstaff, AZ 86001 USA \\ W. M. Farrell ${ }^{1}$ \\ NASA Goddard Space Flight Center, Greenbelt, MD 20771 USA \\ and \\ D. L. Blank \\ Centre for Astronomy, School of Mathematical and Physical Sciences, James Cook University, Townsville \\ QLD 4811, Australia
}

\begin{abstract}
This paper reports Very Large Array observations at 325 and $1425 \mathrm{MHz}(\lambda 90 \mathrm{~cm}$ and $\lambda 20 \mathrm{~cm})$ during and near the periastron passage of HD 80606b on HJD 2454424.86 (2007 November 20). We obtain flux density limits $(3 \sigma)$ of $1.7 \mathrm{mJy}$ and $48 \mu \mathrm{Jy}$ at 325 and $1425 \mathrm{MHz}$, respectively, equivalent to planetary luminosity limits of $2.3 \times 10^{24} \mathrm{erg} \mathrm{s}^{-1}$ and $2.7 \times 10^{23} \mathrm{erg} \mathrm{s}^{-1}$. Unfortunately, these are several orders of magnitude above the nominal Jovian value (at $40 \mathrm{MHz}$ ) of $2 \times 10^{18} \mathrm{erg} \mathrm{s}^{-1}$. The motivation for these observations was that the planetary magnetospheric emission is driven by a stellar wind-planetary magnetosphere interaction so that the planetary luminosity would be elevated near periastron. We estimate that, near periastron, HD 80606b might be as much as 3000 times more luminous than Jupiter. Recent transit observations of HD 80606b provide reasonably stringent constraints on the planetary mass and radius, and, because of the planet's highly eccentric orbit, its rotation period is likely to be "pseudo-synchronized" to its orbital period, allowing a robust estimate of the former. Consequently, we are able to make relatively robust estimates of the emission frequency of the planetary magnetospheric emission and find it to be around $60-90 \mathrm{MHz}$. While this is too low for our reported observations, we compare HD 80606b to other high-eccentricity systems and assess the detection possibilities for both near-term and more distant future systems. Of the known high eccentricity planets, only HD 80606b is likely to be detectable, as the others (HD 20782B and HD 4113) are both lower mass and longer rotational periods, which imply weaker magnetic field strengths. We find that both the forthcoming "EVLA low band" system, which will operate as low as $65 \mathrm{MHz}$, and the Low Frequency Array may be able to improve upon our planetary luminosity limits for HD 80606b, and do so at a more optimum frequency. If the low-frequency component of the Square Kilometre Array (SKA-lo) and a future lunar radio array are able to approach their thermal noise limits, they should be able to detect an HD 80606b-like planet, unless the amount by which the planet's luminosity increases is substantially less than the factor of 3000 that we estimate; for the SKA-lo, which is to be located in the southern hemisphere, future planetary surveys will have to find southern hemisphere equivalents of HD 80606b.
\end{abstract}

Subject headings: planetary systems — planets and satellites: magnetic fields — planets and satellites (HD 80606b) — radio continuum: planetary systemk 


\section{Introduction}

HD 80606 is a G5 star, at a distance of 58.4 pc, which is distinguished by being orbited by a planet with one of the highest known orbital eccentricities. The planet is a giant planet $\left(3.94 \pm 0.11 M_{J}\right)$ with a 111-day orbital period on an extremely eccentric orbit $(e=0.93)$ Naef et al. 2001; Pont et al. 2009).

All of the giant planets in the solar system and the Earth generate radio emission as a result of an interaction between the solar wind and the planetary magnetosphere. Specifically, the solar wind impinging on the planetary magnetosphere generates currents within the magnetosphere which are then directed into the planet's magnetic polar regions where an electron cyclotron maser instability is produced. Motivated by the radio emission from solar system planets, there have been a number of searches for magnetospherically generated radio emission from extrasolar planets, at frequencies ranging from $25 \mathrm{MHz}$ to $1400 \mathrm{MHz}$ (Yantis et al. 1977; Winglee et al. 1986; Bastian et al. 2000; Zarka et al.|2001; Lazio et al. 2004; Rvabov et al. 2004; George \& Stevens 2007; Lazio \& Farrell 2007; Smith et al. 2009; Lecavelier Des Etangs et al. 2009; Lazio et al. 2010). Sensitivities $(1 \sigma)$ have ranged from roughly $1000 \mathrm{mJy}$ to below $1 \mathrm{mJy}$, with generally better sensitivities obtained at the higher frequencies. Typically, these sensitivities have not been sufficient to challenge the predicted levels of extrasolar planetary magnetospheric emission, though some recent multi-epoch $74 \mathrm{MHz}$ observations of $\tau$ Boo (Lazio \& Farrell 2007) do indicate that its planet's emission is not consistent with the most optimistic predictions, unless the emission is either at a frequency well below $74 \mathrm{MHz}$ or is much more strongly beamed than that of solar system planets.

Most recent radio observations of planets have focused on planets in orbits with relatively small semi-major axes and low eccentricities (but see Lazio et al. 2010). Part of the motivation for this focus is the stellar wind powering of the electron cyclotron maser instability. Close to the host star, the stellar wind should be more intense with a con-

\footnotetext{
${ }^{1}$ NASA Lunar Science Institute, NASA Ames Research Center, Moffett Field, CA 94035, USA

${ }^{2}$ current address: Jet Propulsion Laboratory, M/S 138308, 4800 Oak Grove Dr., Pasadena, CA 91109 USA
}

comitant increase in the strength of the planetary radio emission. A secondary cause is the result of a selection effect. Planets close to their host stars produce the largest radial velocity signature, and the vast majority of known extrasolar planets have been found with the radial velocity method.

A concern with this focus on so-called hot Jupiters is that they are likely to be tidally locked to their host star, with rotation periods of order a few days. To the extent that rapid rotation may be necessary for the generation of a strong planetary magnetic field, these "hot Jupiters" may have weak fields that do not generate much electron cyclotron maser emission or generate it at a frequency well below that accessible from the ground. Absent a direct radio detection of magnetically generated emission, evidence for extrasolar planetary magnetic fields is ambiguous. Consistent with the scenario of weak fields, France et al. (2010) were unable to find any unambiguous signal of aurorally generated UV emission from the "hot Jupiter" HD 209458b. From this they derive a limit on the intrinsic planetary magnetic moment of $0.01 \mathcal{M}_{\mathrm{J}}$, but perhaps as large as $0.1 \mathcal{M}_{\mathrm{J}}$, if the interaction with the stellar magnetic field is important. In turn, this limit implies a magnetic field strength of only $10 \%$ that of Jupiter. Batygin \& Stevenson (2010) argue that Ohmic dissipation could explain the inflated atmospheres of "hot Jupiters," and they are able to explain the atmospheres of HD 209458b, HD 189733b, and Tres-4b assuming a field strength of $10 \mathrm{G}$ at the cloud tops, as compared to the value of $4.2 \mathrm{G}$ for Jupiter. Strictly, their estimate $(\sim 10 \mathrm{G})$ is an upper limit because Ohmic dissipation need not be the only effect operating to inflate "hot Jupiter" atmospheres. Lai et al. (2010) show that the asymmetric transit properties of WASP-12b might be able to be explained by a magnetopause, if the planetary magnetic field strength is of order $1 \mathrm{G}$, but their model also has a fairly weak dependence on the magnetic field strength $\left(\propto B^{1 / 3}\right)$. Conversely, a small number of stars with "hot Jupiters" show evidence for star-planet interactions (e.g., Shkolnik et al. 2008), which appear to have a magnetic component. In contrast to "hot Jupiters," with its large eccentricity orbit, HD 80606b may be immune to some of the effects that might act to damp the magnetic field strength of "hot Jupiters." 
The planet orbiting HD 80606 passed through periastron on HJD 2454424.86 (2007 November 20 at 08:05 UT). This paper reports observations at 325 and $1425 \mathrm{MHz}(\lambda 92 \mathrm{~cm}$ and $\lambda 21 \mathrm{~cm}$, respectively) of HD $80606 \mathrm{~b}$ close to its periastron. In Section 2 we describe the observations, analyses, and present the images obtained; in Section 3 we discuss our upper limits in the context of recent multi-wavelength observations of HD 80606b, and in Section 4 we make predictions about future radio observations.

\section{Observations}

HD 80606 was observed 11 at 325 and $1425 \mathrm{MHz}$ with the Very Large Array (VLA), in its B configuration, near the periastron passage of the planet. Table 1 summarizes relevant details of the observations, with specific information about the actual times of observations and the planetary phase. In addition to HD 80606, at both frequencies, 3C 286 was observed to serve as a flux density and bandpass calibrator, and the VLA calibrator J0929+502 was observed as a visibility phase calibrator. Although the VLA could operate at $74 \mathrm{MHz}$ (Kassim et al. 2007), the $74 \mathrm{MHz}$ dipoles were not mounted at the time of these observations.

Standard data reduction techniques were used within the AIPS2 2 software package to calibrate the spectral bandpass, the visibility amplitudes, and visibility phases. The data were acquired in a spectral-line mode both to enable the identification and excision of radio frequency interference (RFI) and for the purposes of wide-field imaging. These issues of RFI excision and wide-field imaging are particularly relevant for the $325 \mathrm{MHz}$ observations.

Following the data calibration, several iterations of imaging and self-calibration were used to produce images of the field around HD 80606; at both frequencies, polyhedral imaging was used to image the primary beam field of view (Cornwell \& Perley 1992). Figures11and2 present the inner portions of the resulting images, centered on HD 80606. The resulting image noise levels are $16 \mu \mathrm{Jy}$ beam $^{-1}$ at $1425 \mathrm{MHz}$ and $580 \mu \mathrm{Jy}_{\text {beam }}{ }^{-1}$

\footnotetext{
1 program AL715.

2 version 31DEC08.
}

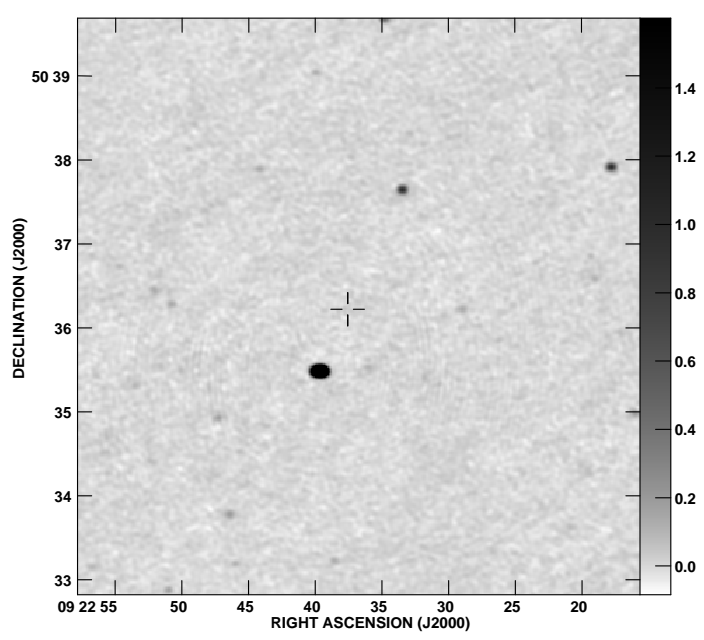

Fig. 1. - Field around HD 80606 at $1425 \mathrm{MHz}$ on HJD 2454425, which includes the time of periastron (Table 1). The cross marks the location of the star. The beam for this image is $5.49 \times 4$ ". 35 , the noise level in the image is $16 \mu \mathrm{Jy}$ beam $^{-1}$, and the gray scale is logarithmic between $-80 \mu \mathrm{Jy}_{\mathrm{beam}}{ }^{-1}$ and $1.6 \mathrm{mJy}^{\text {beam }^{-1}}$. The source to the southeast of the star is FIRST J092239.6+503529.

at $325 \mathrm{MHz}$, which in both cases are within approximately $50 \%$ of the expected thermal noise levels.

\section{Discussion}

At neither frequency do we detect statistically significant emission from the direction of the star. Table 2 summarizes the resulting flux density limits on this system near periastron passage and the resulting luminosity limits. For reference, the nominal luminosity of Jupiter is $2 \times 10^{18} \mathrm{erg} \mathrm{s}^{-1}$ at a frequency near $40 \mathrm{MHz}$.

The age of HD 80606 is uncertain, with estimates ranging from as little as $0.3 \mathrm{Gyr}$ to in excess of 14 Gyr (Wright et al. 2004; Saffe et al. 2005; Valenti \& Fischer 2005; Takeda et al. 2007; Holmberg et al. 2009), but with many estimates favoring an age of approximately 7 Gyr. For the purposes of this discussion, we take the age of HD 80606 to be comparable to that of the Sun, with a comparable stellar wind strength. Consequently, we assume that a Jovian planet, with an orbital semi-major axis comparable to that of 
TABLE 1

OBSERVATION LOG

\begin{tabular}{lcccc}
\hline \hline Frequency & Wavelength & Bandwidth & Epoch & Orbital Phase \\
\hline $325 \mathrm{MHz}$ & $90 \mathrm{~cm}$ & $12.5 \mathrm{MHz}$ & HJD 2454423.89-2454424.22 & $0.69-0.73$ \\
$1425 \mathrm{MHz}$ & $20 \mathrm{~cm}$ & $50 \mathrm{MHz}$ & $\begin{array}{c}\text { 2007 November 19 09:20-17:17 UT) } \\
\text { HJD 2454424.74-2454425.07 } \\
(\text { 2007 November 20 05:46-13:44 UT) }\end{array}$ & $0.94-0.12$ \\
\hline
\end{tabular}

TABLE 2

LUMINOSITY LiMITS FOR HD 80606B

\begin{tabular}{lcc}
\hline \hline Wavelength & $\begin{array}{c}\text { Flux Density Limit } \\
(3 \sigma)\end{array}$ & $\begin{array}{c}\text { Luminosity Limit } \\
\left(\mathrm{erg} \mathrm{s}^{-1}\right)\end{array}$ \\
\hline $1425 \mathrm{MHz}$ & $48 \mu \mathrm{Jy}$ & $2.7 \times 10^{23}$ \\
$325 \mathrm{MHz}$ & $1.7 \mathrm{mJy}$ & $2.3 \times 10^{24}$ \\
\hline
\end{tabular}

Note.-In converting from flux density to luminosity, an emission bandwidth of order the observing frequency is assumed.

Jupiter $(\approx 5 \mathrm{AU})$, would have a radio luminosity similar to that of Jupiter.

It is clear that our luminosity limits remain several orders of magnitude $\left(10^{5}-10^{6}\right)$ above the luminosity of Jupiter. The original motivation for these observations was the expectation that the high eccentricity of the planet's orbit could produce a dramatic variation in the strength of the planet's radio emission. Because a planet's magnetospheric emission is controlled by the input stellar wind power, the luminosity of the planet is a strong function of its distance from the star. Farrell et al. (1999, Appendix A) discuss the scaling of the luminosity with distance and find that an approximate scaling law is $d^{x}$, with $x \approx-1.6$. Griessmeier et al. (2007b,a) consider more complex stellar wind models, but a strong dependence on the planet-star distance remains.

Over the course of the planet's orbit, from apastron to periastron, we therefore expect that the luminosity of HD 80606b could vary by a factor of 200. Even at apastron (0.87 AU), HD 80606b is still closer to HD 80606 than Jupiter is to the
Sun. If we consider the further boost that could be obtained by moving a Jovian-like planet from a distance of $5 \mathrm{AU}$ to $0.87 \mathrm{AU}$, the boost would be a factor of 16 . Thus, one might expect that, near periastron, HD $80606 \mathrm{~b}$ could be as much as a factor of 3000 times more luminous than Jupiter. However, even an increase by a factor of order 3000 over Jupiter's luminosity would be insufficient to increase HD 80606b to a level comparable to our detection thresholds.

A simple explanation for our non-detections is therefore that, even with the added boost due to the small planet-star distance during periastron, the planet's luminosity remains too low to be detected with current equipment. There are several other factors, however, that could also explain our non-detection.

One possibility is that the radio emission is not beamed in our direction. In the case of Jupiter, Zarka et al. (2004) determined that it emits into a solid angle of approximately $1.6 \mathrm{sr}$, directed roughly along Jupiter's rotational axis. HD 80606b is one of the rapidly growing class of 


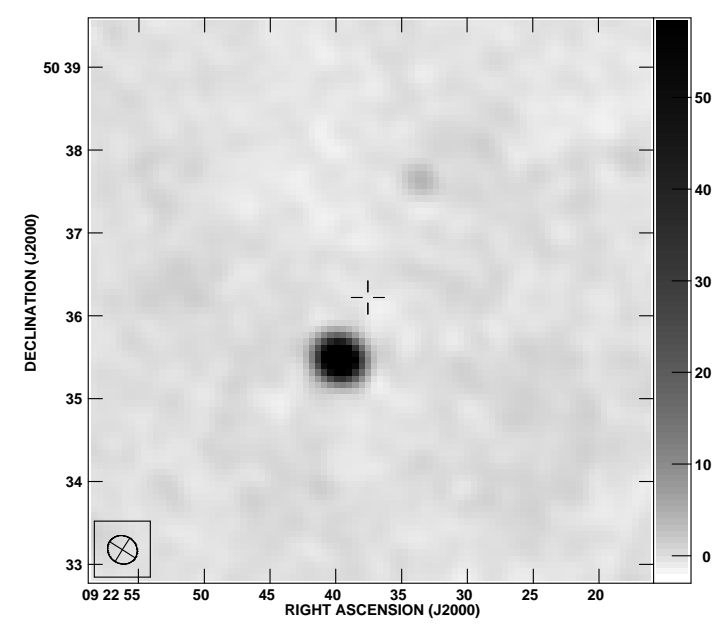

Fig. 2.- Field around HD 80606 at $325 \mathrm{MHz}$ on HJD 2454424, just before the planet's periastron passage (Table 11). The cross marks the location of the star. The beam for this image is $22^{\prime \prime} 1 \times 20^{\prime \prime} 1$ and is shown in the lower left corner; the noise level in the image is $580 \mu \mathrm{Jy}_{\text {beam }^{-1}}$; and the gray scale is logarithmic between $-2.3 \mathrm{mJy}$ beam $^{-1}$ and $58 \mathrm{mJy}^{\text {beam }}{ }^{-1}$. The area shown is approximately the same size as that shown in Figure 1.

transiting extrasolar planets, and recent transit observations demonstrate that there is a significant spin-orbit misalignment $\left(\sim 50^{\circ}\right)$ in this system (Fossev et al. 2009; Garcia-Melendo \& McCullough 2009; Moutou et al. 2009; Pont et al. 2009; Winn et al. 2009). For a transiting planet whose rotational axis is aligned with the stellar rotation axis and no spin-orbit misalignment, the probability of intercepting the emission beam would be relatively small. However, the existing spin-orbit misalignment in this system increases the probability that emission beam intersects the line of sight. Of course, it is possible that the emission cone of HD 80606b is much smaller than that of Jupiter, such that the probability of intercept remains quite low.

A second possibility is that the expected upper frequency limit of emission is (well) below our observation frequencies. The intense cyclotron maser emission of Jupiter cuts off dramatically at frequencies above approximately $40 \mathrm{MHz}$, as the local plasma frequency in the polar emitting region exceeds the electron cyclotron frequency and the radio emission cannot escape. For most extrasolar planets, one can estimate the upper frequency limit by scaling their magnetic moments from that of Jupiter. Previous estimates for the magnetic moment, and upper frequency limit, for HD 80606b have varied wildly. Lazio et al. (2004) estimated an upper frequency limit of around $180 \mathrm{MHz}$, while Griessmeier et al. (2007b) estimated $0.8 \mathrm{MHz}$.

The combination of the eccentric orbit and the planetary transits, however, now provide sufficient information to estimate, with relatively low uncertainty, the upper emission frequency. This upper emission frequency is given by (Farrell et al. 1999; Lazio et al. 2004)

$$
\nu_{\mathrm{c}} \approx 24 \mathrm{MHz}\left(\frac{\omega}{\omega_{\mathrm{J}}}\right)\left(\frac{M}{M_{\mathrm{J}}}\right)^{5 / 3}\left(\frac{R}{R_{\mathrm{J}}}\right)^{3}
$$

for a given planetary mass $M$, rotation and radius $R$. In practice, this value may underestimate the actual upper emission frequency, as the case for Jupiter is $\nu_{\mathrm{c}} \approx 40 \mathrm{MHz}$ whereas Equation (10) suggests $24 \mathrm{MHz}$. This expression also assumes that the planetary magnetic dipole is not offset significantly (as it is for the planets Uranus and Neptune), which can produce significant asymmetric emission.

Of the various determinations of the mass and radius of the planet (Fossev et al. 2009; Garcia-Melendo \& McCullough 2009; Moutou et al. 2009; Pont et al. 2009; Winn et al. 2009), we adopt those that Pont et al. (2009) obtained from a Bayesian analysis of all of the data available to them. They found a planetary mass of $3.94 \pm 0.11 M_{\mathrm{J}}$ and a radius of $0.98 \pm 0.03 R_{\mathrm{J}}$, implying a density about four times higher than that of Jupiter; their models also suggest that the core mass of HD $80606 \mathrm{~b}$ could be several times that of Jupiter.

Hut (1981) discusses the tidal effects in eccentric systems. The large eccentricity for the orbit of HD 80606b should have driven it into a state of pseudo-synchronization between its rotation and orbital periods. Using expressions derived by Hut (1981), we estimate a (pseudo-synchronized) rotational period of $39.9 \mathrm{hr}$.

Evaluating Equation (1), we find that the upper emission frequency of approximately $55 \mathrm{MHz}$, and potentially as high as $90 \mathrm{MHz}$, if one accounts for 
the $50 \%$ difference between the actual value for Jupiter and that predicted by Equation (11). It is clear that this upper emission frequency is well below that of our observations.

This estimate for the emission frequency differs from that of Lazio et al. (2004) by only a factor of $2-3$, but it differs from that obtained by Griessmeier et al. (2007b) by a factor of order $10^{2}$. We believe that the differences can be attributed to assumptions of the rotation period. Lazio et al. (2004) assumed a rotation period equal to that of Jupiter, which is within a factor of 4 of the rotation period we adopt here. However, Griessmeier et al. (2007b) identified the planet as "tidally locked," which would mean that it would have an extremely slow rotation rate, and therefore a low magnetic moment and low emission frequency. One of the key factors that may have led Griessmeier et al. (2007b) to identify the planet as tidally locked is that they appear to have used expressions for estimating the synchronization time scale which assume a circular orbit (their Appendix B).

\section{Future Prospects}

We consider the possibilities of future observations from two perspectives. First, are there planets other than HD 80606b that should be targeted? Second, what are the prospects for future instruments to detect HD 80606b?

While, HD $80606 \mathrm{~b}$ is not the only known planet with a high-eccentricity orbit, it appears the most promising for future radio observations. HD $20782 \mathrm{~b}$ is a $1.9 M_{\mathrm{J}}$ (minimum mass) planet in an orbit with an eccentricity of 0.97 and an orbital period of 592 days (O'Toole et al. 2009), and $\mathrm{HD} 4113 \mathrm{~b}$ is a $1.6 M_{\mathrm{J}}$ (minimum mass) planet in an orbit with an eccentricity of 0.903 and an orbital period of 527 days (Tamuz et al. 2008). The pseudo-synchronization rotational period is coupled to the orbital period, so these longer orbital periods imply substantially larger rotational periods, as compared to HD 80606b. We estimate rotational periods of $63.8 \mathrm{hr}$ and 14 days for HD 20782b and HD 4113b, respectively. Combined with their lower masses, Equation (11) suggests that their emission frequencies are likely to be below $20 \mathrm{MHz}$. Unless future surveys reveal planets with masses and orbital eccentricities comparable to those of HD 80606b (but see
O'Toole et al. 2009), it appears likely to remain the most promising target for radio observations of (high-eccentricity) planets.

With respect to observations of HD $80606 \mathrm{~b}$, our analysis suggests that future observations must be both more sensitive and at a lower frequency than those that we report here. There are several observing systems that are either under construction or in the design phase that we now consider.

The Expanded Very Large Array (EVLA) project exposed certain shortcomings of the VLA's $74 \mathrm{MHz}$ system (Kassim et al. 2007), primarily its generation of harmonics at higher frequencies that fall within the EVLA's operational spectral window. Consequently, the NRL has funded the National Radio Astronomy Observatory to develop a new suite of low-frequency receivers. With the previous $74 \mathrm{MHz}$ VLA system, automated data processing of approximately a $1 \mathrm{hr}$ observation could obtain rms intensity levels of order $100 \mathrm{mJy} \mathrm{beam}^{-1}$ (Cohen et al. 2007), while more focused efforts on longer observations could obtain noise levels of $25 \mathrm{mJy}^{\text {beam }}{ }^{-1}$ Cohen et al. 2004). At a minimum, the new "EVLA-low band" system will have a substantially larger bandwidth. There has also been continuing algorithmic improvements in the areas of RFI identification and excision and ionospheric calibration (e.g., Athreva 2009; Intema et al. 2009; Offringa et al. 2010). As an ansatz, we assume that a combination of algorithmic improvements and the larger bandwidth could produce an approximate factor of 10 improvement in sensitivity, i.e., $3 \mathrm{mJy} \mathrm{beam}^{-1}$. The resulting $(3 \sigma)$ luminosity limit would then be $10^{23} \mathrm{erg} \mathrm{s}^{-1}$ (for an assumed $10 \mathrm{MHz}$ intrinsic emission bandwidth of the electron cyclotron maser emission). This value is comparable to our more stringent limit (at $1425 \mathrm{MHz}$ ), but it would be at a frequency at which emission might more likely be detected.

The Low Frequency Array (LOFAR 3 ) is currently under construction in the Netherlands and other European countries. When complete, it will consist of many dipole phased-array stations, operating in a "high band" (HBA) and a "low band" (LBA). LOFAR-LBA band will cover the frequency range $30-80 \mathrm{MHz}$, with a peak sensitivity around $60 \mathrm{MHz}$, well matched to the ex-

3 http://www.astron.nl/radio-observatory/astronomers/lofar-astronomers 
pected emission frequency of HD 80606b. Current estimates for the sensitivity of LOFAR-LBA are that it will produce a similar or slightly better thermal noise level (about $7 \mathrm{mJy}^{\text {beam }}{ }^{-1}$ in a $1 \mathrm{hr}$ integration) as the EVLA-low band system. We emphasize that the limiting factor in imaging sensitivity might very well not be the thermal noise level, but other factors such as ionospheric calibration. Nonetheless, it appears that the LOFAR-LBA band system would produce similar constraints.

Following the EVLA-low band, LOFAR, and other pathfinder systems are likely to be the lowfrequency component of the Square Kilometre Array (SKA-lo) and ultimately a low frequency array on the Moon (the Lunar Radio Array). The current design goal for SKA-lo has a lower operational frequency of $70 \mathrm{MHz}$ with enough collecting area that the notional thermal noise level would be of order $10 \mu \mathrm{Jy}$ beam $^{-1}$ in a $1 \mathrm{hr}$ integration. Unfortunately, candidate sites for the SKA-lo are in the southern hemisphere (Australia and South Africa, terrestrial latitude $\approx-30^{\circ}$ ), from which HD 80606b cannot be observed. If future planetary surveys detect other HD 80606blike planets and if the thermal noise level can be approached with the SKA-lo, the implied luminosity limit would then be $3 \times 10^{20} \mathrm{erg} \mathrm{s}^{-1}$, well within the range we estimate might be plausible based on the larger stellar wind loading of HD 80606b's magnetosphere.

We thank G. Laughlin for the initial inspiration for these observations and for pointing out that HD 80606b should be in a state of pseudosynchronization; J. Schneider for the Extrasolar Planets Encyclopedia; and P. Perley and W. Koski for helpful discussions. T.J.W.L. thanks USNO, where much of this paper was written, for its hospitality. The National Radio Astronomy Observatory is a facility of the National Science Foundation operated under cooperative agreement by Associated Universities, Inc. The LUNAR consortium is funded by the NASA Lunar Science Institute (via Cooperative Agreement NNA09DB30A) to investigate concepts for astrophysical observatories on the Moon. Basic research at NRL is supported by 6.1 Base funding.

\section{REFERENCES}

Athreya, R. 2009, ApJ, 696, 885

Bastian, T. S., Dulk, G. A., \& Leblanc, Y. 2000, ApJ, 545, 1058

Batygin, K., \& Stevenson, D. J. 2010, ApJ, 714, L238

Cohen, A. S., Röttgering, H. J. A., Jarvis, M. J., Kassim, N. E., \& Lazio, T. J. W. ApJS, 150, 417

Cohen, A. S., Lane, W. M., Cotton, W. D., et al. 2007, AJ, 134, 1245

Cornwell, T. J., \& Perley, R. A. 1992, A\&A, 261, 353

Farrell, W. M., Desch, M. D., \& Zarka, P. 1999, J. Geophys. Res., 104, 14025

Fossey, S. J., Waldmann, I. P., \& Kipping, D. M. 2009, MNRAS, 396, L16

France, K., Stocke, J. T., Yang, H., et al. 2010, ApJ, 712, 1277

Garcia-Melendo, E., \& McCullough, P. R. 2009, ApJ, 698, 558

George, S. J., \& Stevens, I. R. 2007, MNRAS, 382, 455

Griessmeier, J.-M., Preusse, S., Khodachenko, M., Motschmann, U., Mann, G., \& Rucker, H. O. 2007a, Planet. Space Sci., 55, 618

Griessmeier, J.-M., Zarka, P., \& Spreeuw, H. 2007b, A\&A, 475, 359

Holmberg, J., Nordström, B., \& Andersen, J. A\&A, 501, 941

Hut, P. 1981, A\&A, 99, 126

Intema, H. T., van der Tol, S., Cotton, W. D., Cohen, A. S., van Bemmel, I. M., \& Röttgering, H. J. A. 2009, A\&A, 501, 1185

Kassim, N. E., Lazio, T. J. W., Erickson, W. C., et al. 2007, ApJS, 172, 686

Lai, D., Helling, Ch., \& van den Heuvel, E P J. 2010, ApJ, 721, 923 
Lazio, T. J. W., Carmichael, S., Clark, J., Elkins, E., Gudmundsen, P., Mott, Z., Szwajkowski, M., \& Hennig, L. A. 2010, AJ, 139, 96

Lazio, T. J. W., \& Farrell, W. M. 2007, ApJ, 668, 1182

Lazio, T. J. W., Farrell, W. M., Dietrick, J., Greenlees, E., Hogan, E., Jones, C., \& Hennig, L. A. 2004, ApJ, 612, 511

Lecavelier Des Etangs, A., Sirothia, S. K., GopalKrishna, \& Zarka, P. 2009, A\&A, 500, L51

Moutou, C., Hébrard, G., Bouchy, F., et al. 2009, A\&A, 498, L5

O’Toole, S. J., Tinney, C. G., Jones, H. R. A., Butler, R. P., Marcy, G. W., Carter, B., \& Bailey, J. 2009, MNRAS, 392, 641

Pont, F., Hébrard, G., Irwin, J. M., et al. 2009, A\&A, 502, 695

Naef, D., Latham, D. W., Mayor, M., et al. 2001, A\&A, 375, L27

Offringa, A. R., de Bruyn, A. G., Biehl, M., Zaroubi, S., Bernardi, G., \& Pandey, V. N. 2010, MNRAS, 405, 155

Ryabov, V. B., Zarka, P., \& Ryabov, B. P. 2004, Planet. Space Sci., 52, 1479

Saffe, C., Gómez, M., \& Chavero, C. 2005, A\&A, 443,609

Shkolnik, E., Bohlender, D. A., Walker, G. A. H., \& Collier Cameron, A. 2008, ApJ, 676, 628

Smith, A. M. S., Collier Cameron, A., Greaves, J., Jardine, M., Langston, G., \& Backer, D. 2009, MNRAS, 395, 335

Takeda, G., Ford, E. B., Sills, A., Rasio, F. A., Fischer, D. A., \& Valenti, J. A. 2007, ApJS, 168, 297

Tamuz, O., Ségransan, D., Udry, S., et al. 2008, A\&A, 480, L33

Valenti, J. A., \& Fischer, D. A. 2005, ApJS, 159, 141

Winglee, R. M., Dulk, G. A., \& Bastian, T. S. 1986, ApJ, 309, L59
Winn, J. N., Howard, A. W., Johnson, J. A., et al. 2009, ApJ, 703, 2091

Wright, J. T., Marcy, G. W., Butler, R. P., \& Vogt, S. S. 2004, ApJS, 152, 261

Yantis, W. F., Sullivan, W. T., III, \& Erickson, W. C. 1977, BAAS, 9, 453

Zarka, P., Cecconi, B., \& Kurth, W. S. 2004, J. Geophys. Res., 109, A09S15

Zarka, P., Treumann, R. A., Ryabov, B. P., \& Ryabov, V. B. 2001, Ap\&SS, 277, 293

This 2-column preprint was prepared with the AAS IATEX macros v5.2. 